\title{
O QUALIS E OS PERIÓDICOS CIENTÍFICOS NA PRODUÇÃO DE CONHECIMENTO EM DOENÇAS TROPICAIS NEGLIGENCIADAS
}

\author{
QUALIS AND SCIENTIFIC JOURNALS IN THE PRODUCTION OF \\ KNOWLEDGE IN NEGLECTED TROPICAL DISEASES
}

\author{
Natanael Sobral ${ }^{\prime}$ \\ Fabio Mascarenhas e Silva² \\ Zeny Duarte ${ }^{3}$ \\ Tonya Azevedo Duarte ${ }^{4}$
}

\begin{abstract}
RESUMO
Analisa 0 Qualis Capes e os periódicos científicos na produção de artigos em Doenças Tropicais Negligenciadas (DTN), considerando pesquisadores vinculados aos Programas de Pós-Graduação (PPG) e Institutos Nacionais de Ciência e Tecnologia (INCT) aderentes às DTN e os servidores da Fundação Oswaldo Cruz (Fiocruz). 0 período selecionado foi 2015 a 2018, incluindo projeções para 2019. Para isto, selecionaram-se 2719 pesquisadores, coletando seus dados a partir da ferramenta ScriptLattes. Os dados bibliométricos com Qualis foram organizados em ferramentas e instrumentos, tais como, Sistema de Descritores em Ciências da Saúde da Biblioteca Virtual em Saúde (DeCS/BVS), The Vantage Point, Planilha de Cálculo, VOSviewer e UCINET \& NetDraw. Como principais resultados verificaram-se: leishmaniose, doença de Chagas e dengue são as doenças com maior cobertura; no aspecto concentração de artigos em uma mesma revista, leishmaniose, hanseníase, doença de Chagas, mordeduras de serpentes e dengue são as que aparecem mais bem colocadas; Plos One, Ciência \& Saúde Coletiva, Plos Neglected Tropical Diseases, Revista da SBMT e Memórias do IOC são os periódicos mais representativos. Há tendência positiva de artigos nos estratos de maior qualidade, negativa nos de qualidade inferior, estabilização na zona neutra, e projeção de crescimento dos "sem Qualis", tomando por base Medicina II.
\end{abstract}

Palavras-chave: Doenças Tropicais Negligenciadas. Qualis periódicos. Revistas científicas. Produção científica.

\section{ABSTRACT}

Analyzes the Qualis Capes and scientific journals in the production of articles on Neglected Tropical Diseases (DTN), considering researchers linked to the Graduate Programs (GP) and National Institutes of Science and Technology (NIST) adhering to the DTN and employees of Fundação Oswaldo Cruz (Fiocruz). The selected period was 2015 to 2018, including projections for 2019. For this, 2719 researchers were selected, collecting their data from the ScriptLattes tool. The bibliometric data with Qualis were organized into tools and instruments, such as the Descritores em Ciências da Saúde da Biblioteca Virtual em Saúde (DeCS/BVS), The Vantage Point, Calculation Worksheet, VOSviewer and UCINET \& NetDraw. The main results were as follows: leishmaniasis, Chagas disease and dengue are the diseases with the highest coverage; in the aspect of concentration of articles in the same journal, leishmaniasis, leprosy, Chagas disease, snake bites and dengue are the ones that appear best placed; Plos One, Ciência \& Saúde Coletiva, Plos Neglected Tropical Diseases, Revista da SBMT and Memórias do IOC are th e most representative journals. There is a positive trend of articles in the strata of higher quality, negative in those of lower quality, stabilization in the neutral zone, and growth projection of the "without Qualis", based on Medicine II.

Keywords: Neglected Tropical Diseases. Qualis journals. Scientific journals. Scientific production.

Artigo submetido em 24/04/2020 e aceito para publicação em 09/07/2020

1 Professor do Instituto de Ciência da Informação. Universidade Federal da Bahia, Brasil. ORCID http://orcid.org/0000-0003-2410494X. E-mail: natanvsobral@gmail.com

2 Professor da Universidade Federal de Pernambuco, Brasil. Bolsista de Produtividade do CNPq. ORCID https://orcid.org/0000-00015566-5120. E-mail: fabiomascarenhas@gmail.com

3 Professora Titular da Universidade Federal da Bahia, Brasil. ORCID https://orcid.org/0000-0003-0365-6905. E-mail: zenydu@gmail. com

4 Professora da Universidade Federal da Bahia, Brasil. ORCID https://orcid.org/0000-0002-0420-7784. E-mail: tonya.duarte@gmail. com 


\section{INTRODUÇÃO}

As Doenças Tropicais Negligenciadas (DTN) são causadas por agentes infecciosos e parasitários e, embora diversificadas do ponto de vista médico, constituem um grupo, uma vez que todas estão associadas à pobreza e sobrevivem melhor em ambientes tropicais, onde tendem a coexistir (ROSA, 2017). Apesar dos distintos graus de contaminação de cada doença, todas seguem um fluxo baseado na tríade epidemiológica: hospedeiro, ambiente e agente, sendo as enfermidades causadas pela interação entre estas três variáveis (DELIBERATO, 2003; GUIMARÃES, 2015).

A Organização Mundial da Saúde (OMS), baseando-se na 10a reunião do Grupo Consultivo Estratégico e Técnico para DTN (Strategic and Technical Advisory Group for Neglected Tropical Diseases) recebeu propostas para a adição de doenças e, de acordo com os procedimentos exigidos, cromoblastomicose e outras micoses profundas, sarnas e outros ectoparasitas, e envenenamento por mordedura de cobra foram adicionados ao portfólio de DTN. Sendo assim, a lista ficou composta com as seguintes doenças: Buruli Ulcer (Úlcera de Buruli), Chagas disease (Doença de Chagas), Dengue and Chikungunya (Dengue e Chikungunya), Dracunculiasis or guinea-worm disease (Dracunculíase ou Infecção pelo Verme da Guiné), Echinococcosis (Equinococose), Foodborne trematodiases (Trematodíase transmitida por alimentos), Human African trypanosomiasis or sleeping sickness (Tripanossomíase Humana Africana ou Doença do Sono), Leishmaniasis (Leishmaniose), Leprosy or Hansen's disease (Hanseníase), Lymphatic filariasis (Filariose Linfática), Mycetoma (Micetoma), Chromoblastomycosis and other deep mycoses (Cromoblastomicose e outras micoses profundas), Onchocerciasis or river blindness (Oncocercose ou Cegueira de Rio), Rabies (Raiva), Scabies and other ectoparasites (Escabiose e outros ectoparasitas), Schistosomiasis (Esquistossomose), Soiltransmitted helminthiases (Helmintíase transmitida pelo solo), Snakebite envenoming (envenenamento por mordedura de cobra), Taeniasis/Cysticercosis (Teníase/Cisticercose), Trachoma and Yaws - Endemic treponematoses (Tracoma e Bouba - Treponematoses Endêmicas) (OMS, 2017).

A configuração mencionada acima é discutida em âmbito acadêmico, tendo em vista que é dissonante de outras classificações estabelecidas na área de Saúde, que consideram, por exemplo, tuberculose, hepatites, malária, entre outras doenças, aptas a integrar o rol da OMS. 0 próprio termo "DTN" é historicamente considerado inadequado em relação a algumas doenças, como no manual de Doenças Tropicais, publicado pela primeira vez em 1898, pelo médico britânico Patrick Manson, que reconhecia que o termo "doenças tropicais" se mostrava "mais conveniente do que preciso". 0 termo 
"tropical", historicamente, tem servido mais a questões ideológicas, associando-se as causas dessas doenças a fatores naturais, quando de fato são mais influenciadas por questões sociais, econômicas ou políticas (WORBOYS, 2013). Também em tom crítico, Camargo (2008), afirma que as doenças humanas, a priori, são tropicais, pelo fato de a espécie humana ter se originado nos trópicos, e, com ela, suas doenças, exceto as adquiridas ao longo de sua história com seus companheiros de jornada como cães, gatos, roedores, aves, e mesmo de parentes próximos, os primatas. Deste modo, na visão do autor, sendo a maioria das doenças enraizadas nos trópicos, é redundante o uso do termo "tropical". De todo modo, nesse artigo, adotar-se-á a terminologia e o portifólio estabelecido pela OMS, tendo em vista sua liderança e autoridade nos assuntos relacionados à Saúde em todo o mundo.

0 Brasil está inserido em circunstâncias que favorecem o desenvolvimento e avanço das DTN, dada a coexistência das variáveis conjunturais, que envolvem, principalmente: diversidade ecológica, clima quente e úmido, condições sociais de pobreza, parte significativa da população em zona rural e políticas descontinuadas no âmbito do saneamento, educação e saúde pública. Diante deste contexto, chama a atenção o papel de destaque do Brasil na produção científica no campo da Medicina Tropical (Medtrop) e sua liderança nas redes de colaboração neste domínio, evidenciando o engajamento dos pesquisadores e das instituições nacionais na busca por soluções para os problemas locais.

Em consultas realizadas ${ }^{5}$ em duas das maiores bases interdisciplinares de informação científica do mundo, adotando-se a estratégia de busca "neglected tropical disease *", encontraram-se, na Web of Science (WoS), publicações em 129 campos e domínios distintos, incluindo Sociologia, Química Orgânica, Imunologia e Parasitologia, este último, campo com maior número de publicações no assunto. Na Scopus, recuperaram-se publicações em 27 campos e domínios do conhecimento científico diferentes, que vão desde Ciências Planetárias, passando por Odontologia, Matemática, Ciências Sociais, Ciências Biológicas, até a Medicina, campo de maior produtividade no assunto. Um artigo da revista britânica "The Economist", intitulado "Go south, young scientist"6 publicado em 6 de janeiro de 2011, destaca que o Brasil é líder mundial em pesquisa sobre Medtrop, contudo, no momento da publicação do mencionado artigo, o país vivenciava uma fase de amplo investimento nas pesquisas científicas, fato diferente da realidade atual. De modo geral, estes dados alinham-se ao ponto de vista clássico de Forattini (1997), quando afirma que os países desenvolvidos não se veem combatendo

$5 \quad$ As referidas buscas foram realizadas em fevereiro de 2019 e consideraram como campos e domínios as categorias da WoS e as áreas da Scopus. Ressalta-se que mudanças na estratégia de busca, considerando os termos específicos que representam as DTN, certamente, mais campos e domínios que estudam a temática seriam evidenciados, o que demonstra a variedade de disciplinas que se interessam, estudam e produzem conhecimento certificado neste assunto.

6 Disponível em: https://www.economist.com/the-americas/2011/01/06/go-south-young-scientist. Acesso em: 02 mar. 2020 
constantemente problemas de saúde tropical, e por isto, a produção de conhecimento no assunto é baixa em relação a outros temas.

Parte importante do sucesso do Brasil na produção de conhecimento em DTN deve-se aos seus pesquisadores e instituições de Educação, Ciência \& Tecnologia. Porém, por vezes, há imperativos agindo sobre a produção científica que favorecem o direcionamento das publicações para temas que, não necessariamente, estejam relacionados de forma direta com o contexto das DTN. Tais imperativos relacionam-se com a dinâmica "regional" versus "global", fortemente relevante no campo da Saúde, pois, como afirma Coimbra Junior (2004), há um dilema na ciência proporcionado pela dicotomia dos interesses "globais" e "regionais". Se por um lado discutir questões locais específicas contribui para o desenvolvimento regional, promovendo o senso de pertencimento e comprometimento de um determinado pesquisador com os problemas do seu país, por outro, evidenciar as questões globais favorece a aceitação das publicações em periódicos científicos editorados em países de primeiro mundo, com alto fator de impacto.

No cerne dessa discussão, o estrato Qualis da Coordenação de Aperfeiçoamento de Pessoal de Nível Superior (Capes) possui um importante papel, afinal, essa classificação tem por objetivo aferir a qualidade dos periódicos científicos e outros tipos de produção, sendo uma ferramenta usada para classificar a produção científica dos Programas de Pós-Graduação Stricto Sensu (PPG). Tal processo foi concebido para atender as necessidades específicas do sistema de avaliação e é baseado nas informações fornecidas por meio da Plataforma Sucupira (BRASIL, 2014).

Admite-se que se a classificação dos periódicos brasileiros se pautar apenas nos índices fornecidos pelas bases de dados internacionais, muitas revistas científicas nacionais teriam seu estrato Qualis prejudicado, tornando-se invisíveis para os pesquisadores que buscam publicar suas pesquisas em veículos bem classificados, por razões institucionais e de avaliação. Por outro lado, no mundo globalizado, importa que as revistas científicas brasileiras busquem melhorar seu desempenho em âmbito internacional, e para isso, a indexação em bases de dados científicas renomadas e a publicação de artigos em idioma estrangeiro, em especial o inglês, são dois importantes caminhos. Deste modo, cumpre ao Qualis o difícil papel de valorizar a escalada internacional das revistas nacionais e a ampliação do impacto desses veículos nos países desenvolvidos, porém, sem deixar de contemplar os periódicos científicos centrados em pesquisas de impacto regional, não participantes das dinâmicas do ecossistema estabelecido pelo mainstream global, capitaneado pelo primeiro mundo. 
Por esse motivo que o novo Qualis ${ }^{7}$, unificado, vem sendo objeto de crítica por pesquisadores da Saúde Coletiva, área afim à Medtrop pelas posições sociais e centradas em questões da pobreza e das minorias. Sobre isso, Camargo Junior avalia o Qualis único como retrocesso (LOBATO, 2019). Rizzotto, Costa e Lobato (2019) afirmam que esse novo modelo trouxe preocupações e manifestações, tanto por parte de instituições e PPG, como entre os editores científicos de revistas do campo da Saúde Coletiva. Certamente, esse debate estende-se a outros campos do saber, seja em torno da aprovação ou rejeição do novo modelo de classificação.

Deste modo, entende-se que o Qualis é um fator indutor da produção de conhecimento no Brasil, influenciando na escolha dos periódicos a submeterem-se artigos e interferindo no desempenho dos PPG em processos avaliativos. No mais, esta ferramenta de avaliação pode fortalecer um comportamento já existente no campo das DTN, onde algumas doenças com impacto local alocam-se melhor em periódicos nacionais específicos devido à falta de interesse de veículos internacionais no tema, produzindo um nicho de periódicos nacionais com baixo estrato Qualis e poucas possibilidades de ascensão, promovendo implicitamente o desinteresse dos pesquisadores em continuarem pesquisando nesses assuntos.

Tal conjuntura desdobra-se em manifestações do efeito Mateus na Ciência, que apregoa o seguinte: "aos que mais têm será dado em abundância e, aos que menos têm, até o que têm Ihes será tirado" (MERTON, 1968), pois ao final, o Qualis, involuntariamente, pode tornar os pesquisadores brasileiros reféns das políticas editoriais de periódicos internacionais e distantes dos problemas locais. Assim, questiona-se neste artigo sobre a disseminação da produção científica brasileira em periódicos científicos e a possibilidade de correlação entre as doenças e o estrato Qualis das publicações, como oportunidade de mensurar temas que possuem acesso maior aos estratos superiores.

Haja vista a discussão proposta, este artigo objetiva analisar o Qualis Capes e os periódicos científicos frente à produção de artigos em DTN, correlacionando-os com as doenças especificadas pela OMS. Compuseram o universo de indivíduos considerados para a coleta de publicações: os pesquisadores vinculados aos PPG e Institutos Nacionais de Ciência e Tecnologia (INCT) aderentes às DTN e os servidores da Fundação Oswaldo Cruz (Fiocruz). 0 período selecionado foi 2015 a 2018, incluindo algumas projeções para o ano 2019.

7 Disponível em: https://www.capes.gov.br/36-noticias/9730-capes-melhora-ferramentas-de-avaliacao-da-pos-graduacao. Acesso em: 02 mar. 2020. 


\section{METODOLOGIA}

A base epistêmica desta pesquisa é a Análise de Domínio, que é uma teoria e uma abordagem para as áreas de $\mathrm{Cl}$ e Organização do Conhecimento. Neste contexto, combinam-se questões de sistemas e processos de organização do conhecimento a partir de uma perspectiva sociológica e epistemológica, enfatizando a importância do conhecimento do assunto (HJØRLAND, 2017).

Parte-se do princípio que existe um amplo conjunto de conhecimentos que um profissional/ pesquisador especialista em informação precisa obter para trabalhar em domínios específicos, como é o caso dos domínios relacionados à área médica. Neste sentido, Hjørland (2002) afirma que a produção de guias de leitura, classificações e tesauros, indexações e recuperação de informações especializadas, estudos de usuários, análises bibliométricas, pesquisas históricas, estudos de documentos e gênero, estudos epistemológicos e críticos, estudos terminológicos e de discurso, estudos de estruturas e instituições em comunicação científica, análise de domínio em cognição profissional e inteligência artificial são abordagens presentes no cotidiano do profissional/pesquisador da informação, especialista em domínios circunscritos. Por este motivo, neste artigo, o grupo de autores explora os recursos de bases de dados, recuperação da informação, bibliometria e tesauros com ênfase na obtenção, processamento, controle terminológico e visualização das informações, no intuito de adquirir conhecimentos específicos sobre um domínio distinto de suas áreas de formação.

As variáveis consideradas nesse estudo são as DTN, os periódicos científicos e seus estratos Qualis, convergindo esses itens para um diálogo próprio da zona de confluência entre Ciência da Informação (Cl) e Medtrop, envolvendo o domínio cientométrico, a quem compete a análise da produção científica e suas propriedades com amparo da Sociologia da Ciência, que enfatiza a descrição e compreensão das dinâmicas e comportamentos próprios dos campos do conhecimento científico.

Os procedimentos metodológicos empreendidos seguiram o fluxo apresentado abaixo:

a) Coleta de Dados: Os atores que compuseram o universo do estudo foram selecionados conforme critério de atuação institucional formal em atividades de pesquisa no campo das DTN, estabelecendo-se três grupos representativos que envolvem: servidores da Fiocruz e indivíduos vinculados a PPG (permanentes, colaboradores e visitantes) e aos INCT.

A Fiocruz ${ }^{8}$ é a principal instituição de pesquisa não-universitária de formação e qualificação de recursos humanos para o Sistema Único de Saúde (SUS) e para a área de C\&T no Brasil. Possui 32

$8 \quad$ Ver detalhes em: https://www.icict.fiocruz.br/pesquisadores-do-instituto 
programas de pós-graduação stricto sensu em diversas áreas, uma escola de nível técnico e vários programas lato sensu (BRASIL, 2019). Dada sua autoridade, vanguarda e intrínseca relação com as questões relacionadas às DTN, a Fiocruz foi selecionada para integrar o universo desta pesquisa. Tal autoridade vem sendo comprovada em diversos estudos, tais como, González-Alcaide et al., (2012); González-Alcaide et al., (2013) e Sampaio (2015). Em linha similar, Sobral, Silva e Duarte (2017) constatam a liderança do Brasil na América Latina em Medtrop, destacando-se o papel da Fiocruz e das Universidades Federais.

A lista dos servidores da instituição foi cedida pelo projeto "e-Lattes: um novo arcabouço em linguagem R para análise do currículo Lattes"9. A planilha obtida, com base no Portal da Transparência e Plataforma Lattes do Conselho Nacional de Desenvolvimento Científico e Tecnológico (PL/CNPq), possui os seguintes campos: ID Lattes, nome do servidor, e-mail, nacionalidade, ingresso na Fiocruz, unidade de lotação, sigla da unidade de lotação, carga horária, escolaridade, cargo, nível do cargo, situação funcional, sexo e status. Apenas os pesquisadores com doutorado foram selecionados, tendo em vista que os pressupostos principais de composição de universo são o envolvimento e a ativa participação na pesquisa científica. No total, 1870 indivíduos foram considerados.

Dados do relatório Research in Brazil - A report for Capes by Clarivate Analytics apontam que mais de $95 \%$ da produção científica do Brasil nas bases internacionais, deve-se à capacidade de pesquisa de suas universidades públicas federais e estaduais (CROSS; THOMSON; SINCLAIR, 2017; UNIFESP, 2019; MOURA, 2019). Por isso, as Universidades, em especial os PPG, cumprem um papel fundamental na Ciência brasileira, sendo parte indispensável do universo desta pesquisa. A estratégia para a identificação dos atores vinculados aos PPG baseou-se na área de Medicina II, que envolve a Medtrop, selecionando-se os programas que possuíam vinculação temática com as DTN. Para esta identificação, buscaram-se os termos: "tropica*", "infec*”, "negligenciada*”, "parasitária*”, no nome do programa, áreas de concentração, áreas básicas e linhas de pesquisa ${ }^{10}$. 0 ano de vínculo oferecido pela Plataforma Sucupira no momento da busca foi 2018 e 555 pesquisadores foram mapeados. Com esta estratégia, identificaram-se os seguintes programas:

9 Disponível em: https://medium.com/data-net-sci/e-lattes-um-novo-arcabou\%C3\%A70-em-linguagem-r-para-an\%C3\%A1lise-do-curr\%C3\%ADculo-lattes-9ecb2f68574e

100 asterisco indica que os termos foram considerados independentemente de suas terminações. 
Tabela 1 - Programas de Pós-Graduação Stricto Sensu que fazem interface com DTN

\begin{tabular}{|c|l|c|c|}
\hline \multicolumn{2}{|c|}{ Nome do Programa } & Instituição & $\begin{array}{c}\text { Qtde. } \\
\text { Pesquisadores }\end{array}$ \\
\hline 1 & Ciências da Saúde na Amazônia Ocidental & UFAC & 23 \\
\hline 2 & Doenças Infecciosas & UFES & 19 \\
\hline 3 & Doenças Infecciosas e parasitárias (Ms. Profissional) & USP & 10 \\
\hline 4 & Doenças Infecciosas e parasitárias & USP & 23 \\
\hline 5 & Medicina (Doenças Infecciosas e Parasitárias) & UFRJ & 20 \\
\hline 6 & Doenças Infecciosas e Parasitárias & UFMS & 20 \\
\hline 7 & Doenças Tropicais & UFPa & 20 \\
\hline 8 & Doenças Tropicais & Unesp/Botucatu & 22 \\
\hline 9 & Pesquisa Clínica & Fiocruz & 48 \\
\hline 10 & Medicina Tropical & Fiocruz & 57 \\
\hline 11 & Infecções, HIV, AlDS e Hepatites Virais & UNIRIO & 45 \\
\hline 12 & Infectologia & UNIFESP & 18 \\
\hline 13 & Infectologia e Medicina Tropical & UFMG & 25 \\
\hline 14 & Medicina Tropical & UFPE & 15 \\
\hline 15 & Medicina Tropical & UEA & 34 \\
\hline 16 & Medicina Tropical e Saúde Pública & UFG & 32 \\
\hline 17 & Medicina Tropical & USP & 30 \\
\hline 18 & Medicina Tropical & UnB & 25 \\
\hline 19 & Medicina Tropical e Infectologia & UFTM & 21 \\
\hline 20 & Saúde na Amazônia & UFPA & 24 \\
\hline 21 & Virologia & UFPa & 24 \\
\hline
\end{tabular}

Fonte: Dados da pesquisa (2020)

Por fim, elegeram-se os grupos atendidos pelo Programa INCT. Segundo o CNPq (2014), 0 programa visa: agregar, de forma articulada, os melhores grupos de pesquisa na fronteira da ciência e em áreas estratégicas para o desenvolvimento sustentável do país; impulsionar a pesquisa científica básica e fundamental competitiva internacionalmente; estimular o desenvolvimento de pesquisa científica e tecnológica de ponta associada a aplicações para promover a inovação e 0 espírito empreendedor. Deste modo, conforme destaca a Estratégia Nacional de Ciência, Tecnologia e Inovação - 2016-2022 (2016), os INCT compõem um programa estratégico que congrega as unidades de pesquisa de maior excelência no país, sendo operadores do SNCTI, tendo como competência a execução das atividades de PD\&I planejadas, ocupando a posição indicada na Figura 1 (BRASIL, 2016). 
Figura 1 - Principais atores do Sistema Nacional de Ciência, Tecnologia \& Inovação
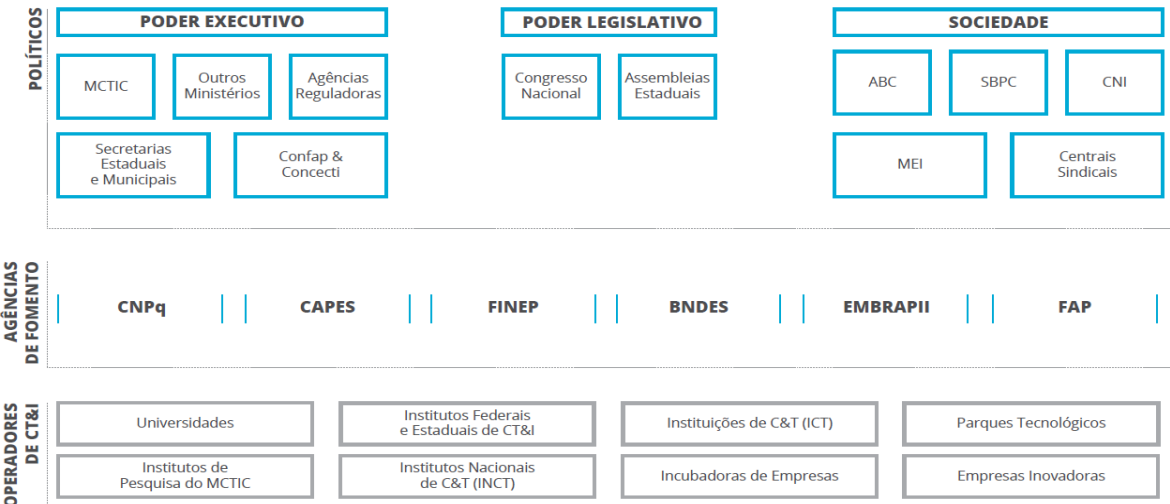

CAPES || FINEP || BNDES

EMBRAPII

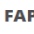

Fonte: Estratégia Nacional de Ciência, Tecnologia e Inovação - 2016-2022 (2016)

Para a seleção dos INCT em destaque no universo desta pesquisa, acessou-se o site do programa ${ }^{11}$, consultando a aba "Institutos", adentrando na categoria "Saúde". Com isto, elegeram-se apenas os INCT, que em sua ementa, afirmam trabalhar com doenças negligenciadas, infecciosas, parasitárias ou tropicais, tendo algumas destas patologias como foco ou interface de pesquisa. Assim, 12 institutos, com um total de 403 pesquisadores foram incorporados ao universo (Tabela 2).

Tabela 2 - INCT com interface em DTN

\begin{tabular}{|r|l|l|l|}
\hline \multicolumn{2}{|c|}{ Sigla } & \multicolumn{1}{c|}{ Nome do INCT } & \multicolumn{1}{c|}{$\begin{array}{c}\text { Qtde. } \\
\text { Pesquisadores }\end{array}$} \\
\hline 2 & CIBFAR & $\begin{array}{l}\text { Centro de Pesquisa e Inovação em Biodiversidade e } \\
\text { Fármacos }\end{array}$ & 22 \\
\hline 3 & INCT-IDN & $\begin{array}{l}\text { Instituto Nacional de Ciência e Tecnologia de Gestão da } \\
\text { Inovação em Doenças Negligenciadas } \\
\text { Instituto Nacional de Ciência e Tecnologia de Biotecnologia } \\
\text { Estrutural e Química Medicinal em Doenças Infecciosas }\end{array}$ & 60 \\
\hline 4 & INOFAR & $\begin{array}{l}\text { Instituto Nacional de Ciência e Tecnologia de Fármacos e } \\
\text { Medicamentos }\end{array}$ & 38 \\
\hline 5 & INCT-DT & $\begin{array}{l}\text { Instituto Nacional de Ciência e Tecnologia em Doenças } \\
\text { Tropicais }\end{array}$ & 16 \\
\hline 7 & INCT-INPeTAm & $\begin{array}{l}\text { Instituto Nacional de Ciência e Tecnologia em Pesquisa } \\
\text { Translacional em Saúde e Ambiente na Região Amazônica }\end{array}$ & 46 \\
\hline 8 & INCT-FHV & $\begin{array}{l}\text { Instituto Nacional de Ciência e Tecnologia para Febres } \\
\text { Hemorrágicas Virais }\end{array}$ & 25 \\
\hline
\end{tabular}

11 Disponível em: http://inct.cnpq.br/ 


\begin{tabular}{|r|l|l|l|}
\hline 9 & INCT-INDI & $\begin{array}{l}\text { Instituto Nacional de Ciência e Tecnologia para Diagnóstico } \\
\text { em Saúde Pública }\end{array}$ & 42 \\
\hline 10 & INCT-iii & $\begin{array}{l}\text { Instituto Nacional de Ciência e Tecnologia de Investigação } \\
\text { em Imunologia }\end{array}$ & 33 \\
\hline 11 & INCT-TB & Instituto Nacional de Ciência e Tecnologia em Tuberculose & 35 \\
\hline 12 & INCT-V & Instituto Nacional de Ciência e Tecnologia de Vacinas & 39 \\
\hline
\end{tabular}

Fonte: INCT (2020)

Na soma, 2828 pesquisadores foram identificados. Ao eliminarem-se as redundâncias, verificou-se um total de 2719 pesquisadores, que efetivamente, tiveram suas produções coletadas e consideradas.

b) Extração de dados dos pesquisadores: Para a realização do levantamento dos dados dos pesquisadores optou-se pela PL/CNPq, valendo-se da ferramenta ScriptLattes (MENA-CHALCO; CÉSAR JUNIOR, 2009). Apesar de utilizar-se da extração completa fornecida pelo software, selecionaram-se, especificamente os registros da produção de artigos de periódicos e o Qualis Capes, consoante ao quadriênio 2013-2016, último disponibilizado na Plataforma Sucupira. 0 período da coleta de dados no ScriptLattes foi 2015 a 2018. Para fins de predição quantitativa de tendências futuras, uma segunda coleta foi realizada, com os mesmos atores e categorias, considerando apenas o ano 2019. A coleta foi executada no início do mês de maio de 2019.

c) Organização e Tratamento da Informação: Essa etapa permitiu o tratamento terminológico de questões ligadas ao idioma e às palavras-chave. 0 objetivo de criar este conjunto de termos foi validar se as publicações científicas dos pesquisadores tratam ou não das doenças negligenciadas. Os nomes das doenças foram as categorias temáticas de análise, estabelecidas conforme 0 site da OMS ${ }^{12}$, a saber: Buruli ulcer, Chagas disease, Dengue and Chikungunya, Dracunculiasis (guinea-worm disease), Echinococcosis, Foodborne trematodiases, Human African trypanosomiasis (sleeping sickness), Leishmaniasis, Leprosy (Hansen's disease), Lymphatic filariasis, Mycetoma, chromoblastomycosis and other deep mycoses, Onchocerciasis (river blindness), Rabies, Scabies and other ectoparasites, Schistosomiasis, Soil-transmitted helminthiases, Snakebite envenoming, Taeniasis/Cysticercosis, Trachoma, Yaws (Endemic treponematoses). Todas as palavras-chave foram obtidas a partir de uma técnica de mineração de textos que fraciona os títulos dos artigos, sendo estes, obtidos a partir do arquivo ris advindo do ScriptLattes. Prontamente, para a padronização dos termos-chave utilizou-

12 Disponível em: https://www.who.int/neglected_diseases/diseases/en/. 
se o Sistema de Descritores em Ciências da Saúde da Biblioteca Virtual em Saúde (DeCS/BVS) ${ }^{13}$, contemplando os sinônimos, descritores em espanhol, inglês e português para que não houvesse dispersão.

Em seguida, utilizou-se 0 software The Vantage Point $\mathbb{R}^{14}$ no intuito de realizar a limpeza dos dados e estandardização; cruzamento de dados (possibilitando o entrelaçamento de campos para análises multivariadas) e a elaboração de matrizes, que determinaram a ausência ou presença de relação entre categorias de dados, como por exemplo: DTN \& Periódicos Científicos e DTN \& Qualis Capes.

d) Análise do Qualis e das Revistas Científicas: A Medtrop está inserida na área de Medicina II na Capes ${ }^{15}$, que é composta pelas seguintes especialidades médicas: 1) Doenças Infeciosas e Parasitárias/Infectologia; 2) Patologia; 3) Pediatria/Saúde da Criança e do Adolescente; 4) Neurologia/ Neurociências; 5) Psiquiatria/Saúde Mental; 6) Radiologia e Diagnóstico por Imagens; 7) Hematologia; 8) Reumatologia; 9) Alergologia. Para o mapeamento do Qualis utilizou-se um arquivo de texto incorporado à ferramenta ScriptLattes, obtido no site da Plataforma Sucupira, inicialmente, como planilha. Esse processo permitiu a identificação de todas as revistas qualificadas em Medicina II, com seus respectivos estratos, processados no ScriptLattes para comparação com as listas de periódicos em que os pesquisadores publicaram.

Também foram verificadas as principais revistas científicas e suas relações com as DTN, reforçando o papel dos periódicos enquanto espaços de alocação e difusão do conhecimento científico produzido sobre o tema, seja em âmbito local, regional, nacional ou internacional. Acredita-se que reconhecer os veículos com alta aceitabilidade às questões de DTN é de fundamental importância para que se direcionem recursos específicos referentes a custos de publicação, tradução, entre outras demandas que ajudem a fortalecer as possibilidades de aceitação das produções, nestes veículos, dos pesquisadores em DTN atuantes no Brasil.

e) Elementos Prospectivos: Haja vista que a data da coleta de dados para este artigo foi início de maio de 2019 e os dados alusivos a este ano refletem apenas o primeiro quadrimestre de 2019, optou-se por analisá-los em separado, apartando-os de 2015 a 2018, anos em que os dados foram obtidos em sua completude.

13 Disponível em: http://decs.bvs.br/.

14 The Vantage Point é uma ferramenta de mineração de texto para descoberta de conhecimento em bases de dados científicas e de patentes. Esta ferramenta possibilita a geração de indicadores científicos a partir de um conjunto de dados bibliométricos oferecidos como entrada (input). Disponível em: https://www.thevantagepoint.com/.

15 Informações extraídas do Documento de Área (Medicina II) - 2016: Disponível em: http://capes.gov.br/images/documentos/Documentos_de_area_2017/16_MED_2_docarea_2016.pdf 
Deste modo, destaca-se que é muito comum os pesquisadores não atualizarem seus currículos Lattes nos primeiros meses do ano, pois, em geral, suas instituições estão de férias ou iniciando o semestre letivo, desenvolvendo prioritariamente atividades de planejamento. Assim, visando se aproximar de parâmetros legítimos para criar uma base comparativa que trouxesse subsídios para predizer o resultado do ano 2019, adotou-se a estratégia a seguir: selecionaram-se as datas de atualização dos currículos Lattes do recorte 2019. Assim, evitou-se a possibilidade de considerarem-se pesquisadores que há muito tempo não atualizam seus currículos. Aplicado este critério, verificouse que a média de atualização de currículos da produção "2019" foi "2 de abril de 2019". Por este motivo, considera-se que a produção concernente a este ano, em média, corresponde a de três meses completos (janeiro, fevereiro e março), constituindo assim, um trimestre. Deste modo, sendo três meses equivalente a um quarto de um ano, a produção foi multiplicada por quatro, obtendo-se assim as projeções.

f) Representação dos dados: No que tange à representação gráfica e as formas de simbolização, utilizaram-se as ferramentas Microsoft Exce/®, com o propósito de observar questões de avanço, retrocesso, dispersão e acumulação presentes na produção científica. Com relação às redes, utilizaram-se as ferramentas VOSviewere UCINET \& NetDraw, estes dois últimos, propostos por Borgatti, Everett e Freeman (2002). Tais ferramentas serviram para a análise do conjunto de relações estabelecidas entre as variáveis, seguindo os princípios metodológicos estabelecidos pelo domínio da Análise de Redes Sociais (ARS), amplamente divulgados por Wasserman e Faust (1994).

\section{RESULTADOS E DISCUSSÃO}

Os resultados estão organizados conforme o seguinte ordenamento: 1) DTN versus revistas científicas, incluindo todas as variáveis; 2) Média de artigos publicados por variedade de revistas científicas; 3) Vínculos mais estreitos entre DTN e revistas científicas; 4) Estrato Qualis Capes versus DTN; 5) DTN distribuídas percentualmente pelo estrato Qualis de suas publicações; e 6) Qualis Capes, ano a ano, com parcial e previsão 2019 (tendências).

Em princípio, apresentam-se as DTN, relacionando-as aos periódicos, objetivando encontrar nichos em revistas científicas que servem ao propósito de "escoar" fluxos de produção científica sobre determinados temas, o que pode ser verificado na Figura 2. 
Figura 2 - Doenças Tropicais Negligenciadas versus revistas científicas, no universo analisado: 2015 a 2018: todas as variáveis

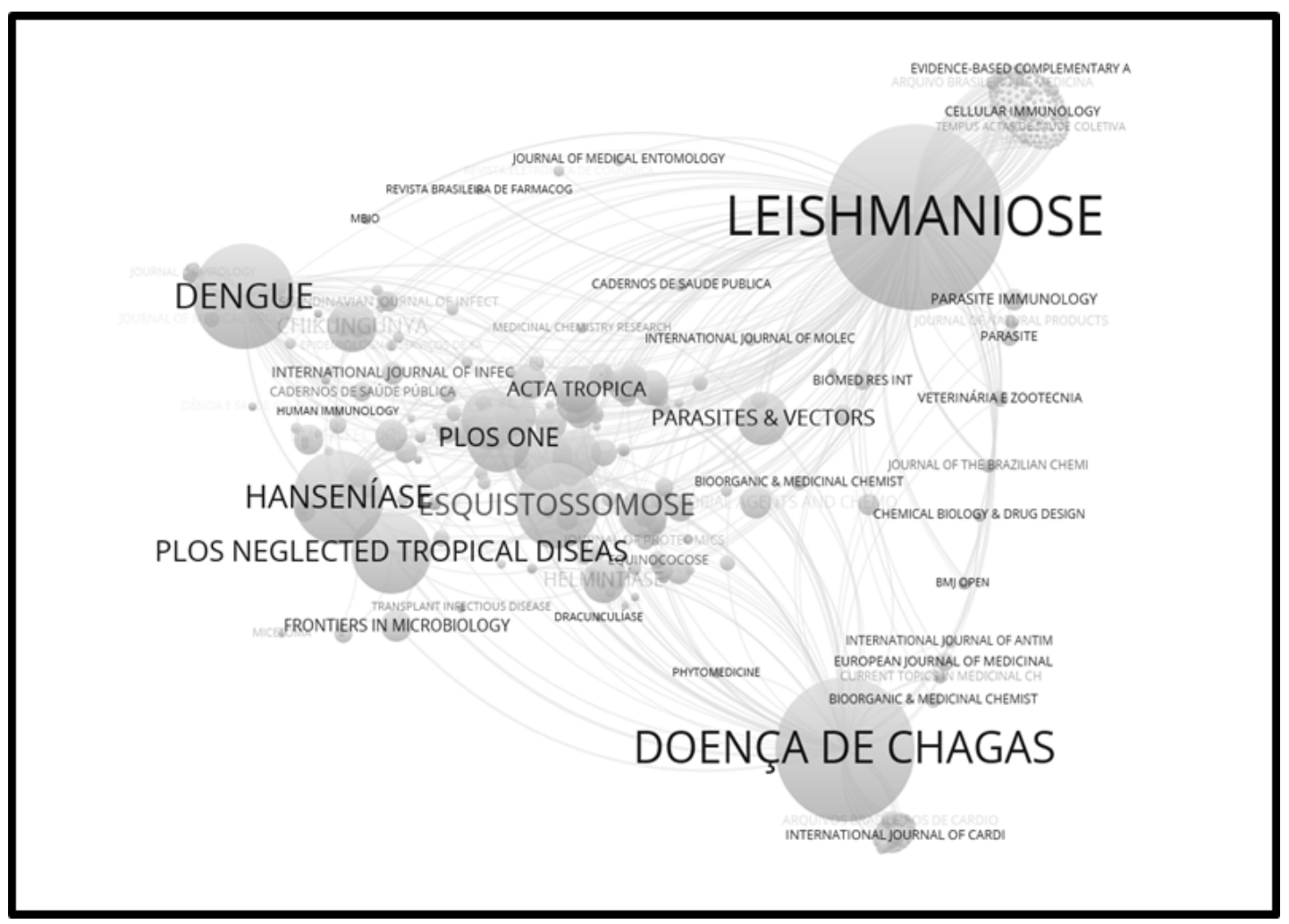

Fonte: Dados da pesquisa (2020)

A Figura 2 destaca os tópicos mais representativos, incluindo doenças, revistas científicas e suas relações. Ao centrar-se a análise nas revistas, nota-se um total de 669 periódicos, sendo que, leishmaniose está presente em 282, doença de Chagas em 237, dengue em 154, esquistossomose em 111, hanseníase em 100, febre de Chikungunya em 41, helmintíase em 38, mordeduras de serpentes em 32, filariose em 24, tracoma em 21, raiva em 14, cisticercose em 11, equinococose em 9, teníase em 8, cromoblastomicose em 6, fasciolíase em 3, dracunculíase em 2, escabiose em 2, micetoma em 2, oncocercose em 1. Ressalta-se que, uma revista pode abrigar mais de um tema, a depender dos artigos que publica, por isso, o número total das ocorrências de doenças supera a quantidade de revistas. Com isto, verifica-se que leishmaniose, doença de Chagas e dengue não são apenas as DTN com maior produção, mas também, com maior cobertura. Hanseníase aparece em quarto lugar no quesito produção de artigos, enquanto esquistossomose é a quinta colocada, todavia, quando se trata de cobertura, esquistossomose aparece à frente de hanseníase. A Tabela 3 apresenta a relação quantidade, cobertura e média de aparição das DTN por revistas. 
Tabela 3 - Média de artigos publicados por variedade de revistas científicas no universo analisado: 2015 a 2018

\begin{tabular}{|l|c|c|c|}
\hline \multicolumn{1}{|c|}{ DTN } & $\begin{array}{c}\text { Artigos } \\
\text { Publicados }\end{array}$ & $\begin{array}{c}\text { Variedade de revistas que } \\
\text { publicam sobre o assunto }\end{array}$ & $\begin{array}{c}\text { Média (Artigos/ } \\
\text { Revistas) }\end{array}$ \\
\hline Leishmaniose & 988 & 282 & 3,5 \\
\hline Hanseníase & 253 & 100 & 2,53 \\
\hline Doença de Chagas & 596 & 237 & 2,51 \\
\hline Mordeduras de Serpentes & 73 & 32 & 2,28 \\
\hline Dengue & 320 & 154 & 2,07 \\
\hline Filariose & 47 & 24 & 1,95 \\
\hline Esquistossomose & 209 & 111 & 1,88 \\
\hline Febre de Chikungunya & 75 & 41 & 1,82 \\
\hline Cisticercose & 20 & 11 & 1,81 \\
\hline Cromoblastomicose & 10 & 6 & 1,66 \\
\hline Teníase & 12 & 8 & 1,5 \\
\hline Helmintíase & 56 & 38 & 1,47 \\
\hline Tracoma & 26 & 21 & 1,23 \\
\hline Raiva & 15 & 14 & 1,07 \\
\hline Equinococose & 9 & 9 & 1 \\
\hline Fasciolíase & 3 & 3 & 1 \\
\hline Dracunculíase & 2 & 2 & 1 \\
\hline Escabiose & 2 & 2 & 1 \\
\hline Micetoma & 1 & 2 & 1 \\
\hline Oncocercose & 2 & 2 & 1 \\
\hline
\end{tabular}

Fonte: Dados da pesquisa (2020)

A Tabela 3, ordenada pela média, indica que no aspecto concentração de artigos em uma mesma revista, leishmaniose, hanseníase, doença de Chagas, mordeduras de serpentes e dengue são as que aparecem mais bem colocadas, sugerindo que estas doenças apresentam maior incidência de artigos em periódicos específicos, que pode ser fruto do perfil temático da revista, do pesquisador que submete frequentemente os manuscritos para estes veículos ou da existência de convocatórias especiais de publicação para determinados temas. Por outro lado, os temas que caminham abaixo da média 2, estão mais pulverizados em periódicos diferentes, com maior possibilidade de dispersão, expressando que o assunto está, percentualmente, distribuído em um quantitativo superior de veículos.

Considerando que a rede DTN versus revistas possui 1096 vínculos, buscou-se verificar quais as relações mais profundas entre estas entidades (Figura 3). 
Figura 3 - Vínculos mais estreitos entre DTN e revistas científicas, no universo analisado:

$$
2015 \text { a } 2018 \text { (relações > 8) }
$$

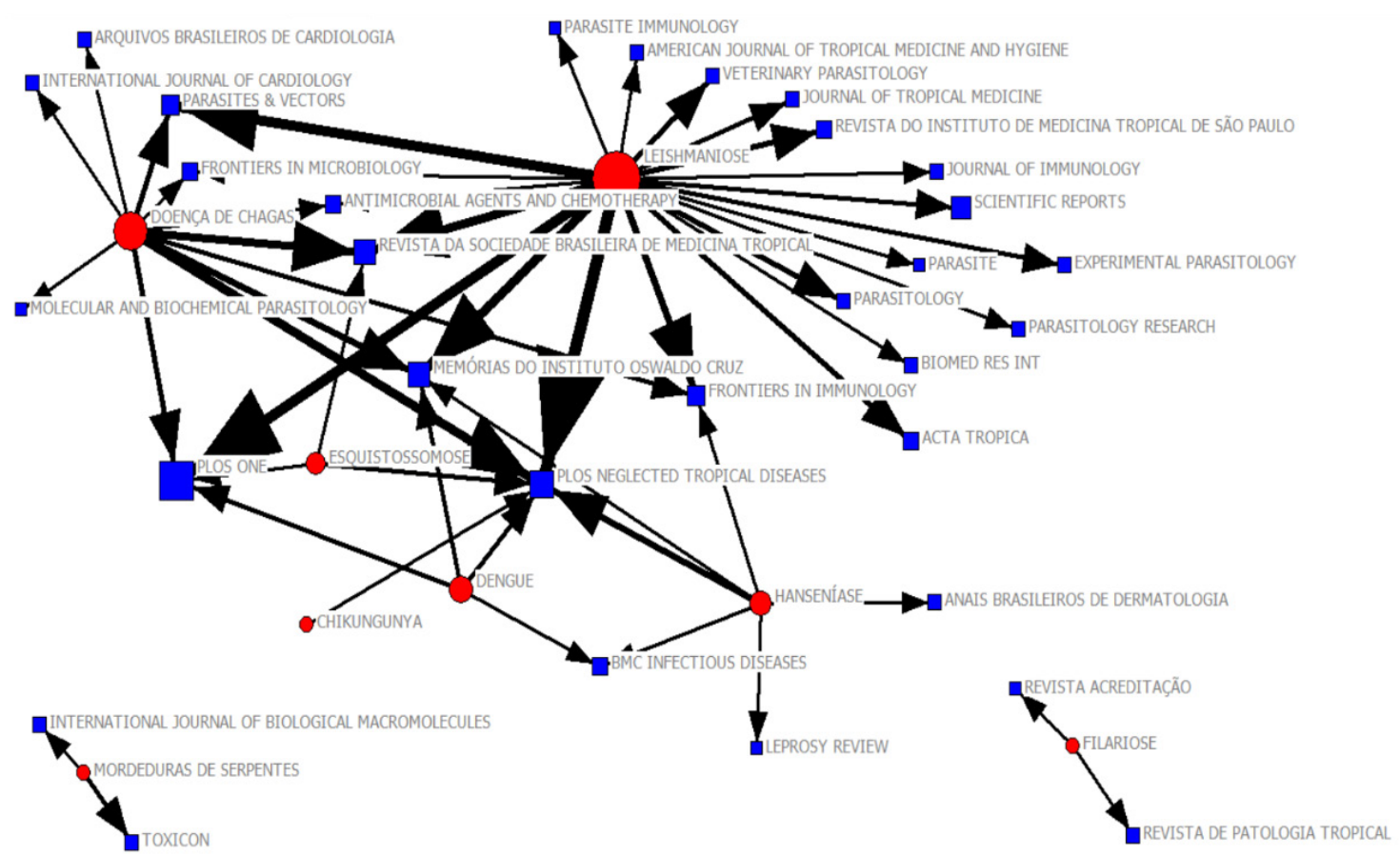

Fonte: Dados da pesquisa (2020)

Identificaram-se os seguintes núcleos relacionais: leishmaniose \& Plos Neglected Diseases (62), leishmaniose \& Plos One (53), leishmaniose \& Parasites \& Vectors (43), doença de Chagas \& Plos Neglected Diseases (41), leishmaniose \& Revista da SBMT (37), leishmaniose \& Memórias do IOC (37), leishmaniose \& Frontiers in Immunology (33), doença de Chagas \& Revista da SBMT (32), doença de Chagas \& Memórias do IOC (29), hanseníase \& Plos Neglected Diseases (29), doença de Chagas \& Plos One (25), leishmaniose \& Revista do Instituto de Medicina Tropical de São Paulo (25), leishmaniose \& Parasitology (25), doença de Chagas \& Parasites \& Vectors (24), leishmaniose \& Acta Tropica (22), leishmaniose \& Veterinary Parasitology (20), dengue \& Plos Neglected Tropical Diseases (19), esquistossomose \& Plos Neglected Tropical Diseases (19), leishmaniose \& Experimental Parasitology (18), doença de Chagas \& Frontiers in Immunology (17), mordedura de Serpentes \& Toxicon (16), dengue \& Memórias do IOC (16), dengue \& Plos One (16), leishmaniose \& Scientific Reports (16).

No aspecto quantitativo, as revistas que mais se destacaram, com mais de 100 registros foram as seguintes: Plos One (603), Ciência \& Saúde Coletiva (337), Plos Neglected Tropical Diseases (316), 
Revista da SBMT (284), Memórias do IOC (281), Cadernos de Saúde Pública (212), Scientific Reports (195), Parasites \& Vectors (178), Frontiers In Immunology (166), Saúde em Debate (151), Revista do Instituto de Medicina Tropical de São Paulo (134), Revista de Saúde Pública (129), Acta Tropica (122), Frontiers in Microbiology (117) e The Brazilian Journal of Infectious Diseases (114).

A partir destes dados é possível ter uma ideia das fontes de informação úteis à consolidação das políticas científicas sobre publicações, haja vista que foram identificados os periódicos preferidos pelos pesquisadores brasileiros sobre temas específicos em DTN. Percebe-se um equilíbrio entre veículos internacionais e nacionais, demonstrando a inserção da área no paradigma global, em que os processos de internacionalização são preconizados, em especial, nas publicações em língua inglesa. E também, ratificando a presença das revistas brasileiras, notadamente, aquelas que são internacionalizadas por estarem indexadas nas grandes bases comerciais de produção científica.

Outro interessante achado refere-se às revistas científicas que possuem maior cobertura temática nos assuntos de interesse deste artigo. Sobre este item, verificaram-se os periódicos que abrangem uma maior quantidade de doenças, tendo como parâmetro o universo selecionado. Assim, constatou-se que: Plos Neglected Tropical Diseases (13), Revista do Instituto de Medicina Tropical de São Paulo (12), Plos One (11), Acta Tropica (11), Revista da SBMT(10), Memórias do IOC (8), Parasites \& Vectors (8), Scientific Reports (7) Journal of Tropical Medicine (7), Cadernos de Saúde Pública (6), Frontiers in Immunology (6), BMC Infectious Diseases (6) e Parasitology (6) são as revistas mais tentacularizadas, abrangendo os temas em DTN de maneira plural e diversificada, e por isso, merecem destaque neste artigo.

Quanto ao Qualis, buscou-se observar a alocação de temas por estrato visando perceber quais DTN conseguem se sobressair em relação a esta classificação de qualidade. Inicialmente, reconhecese que há várias motivações e critérios para que um artigo seja considerado, aprovado e publicado em um periódico bem classificado, incluindo: a) qualidade do manuscrito; b) padronização alinhada às normas técnicas vigentes e às diretrizes específicas do periódico; c) relevância e originalidade; d) rigor teórico e metodológico; e) adequação ao escopo temático do veículo; f) redação e organização do texto; g) qualificação e autoridade dos autores; h) sistema de avaliação adotado e acurácia dos avaliadores; i) pagamento de taxas de publicação, quando se aplica; j) atendimento, por parte dos autores, aos prazos de devolutiva e ajustes do artigo; entre outros. Ademais, há de se considerar que o estrato dos periódicos científicos é uma variável mutável e circunstancial, portanto, numa avaliação uma revista pode receber classificação alta e na seguinte, sofrer uma redução do seu estrato. Do mesmo modo, 0 contrário também pode ocorrer. 
Desta feita, não se pode assumir que o tema de um artigo é uma variável peremptória para 0 acesso a estratos específicos do Qualis. Ainda assim, reconhece-se o capital diferenciado que alguns temas recebem da comunidade científica, visível nas políticas explícitas e implícitas dos periódicos mais relevantes, seja pela sua importância para um determinado momento ou problema da sociedade ou pela sua valia para grupos de pesquisadores e/ou entidades. Por isso, entende-se que esse sistema científico apesar de rigoroso e orientado à qualidade, dada sua característica social, também é composto por privilégios, predileções e inclinações. Carregam indícios disto, as políticas editoriais dos veículos, pois objetivamente determinam os assuntos passíveis de aceitação. Subjetivamente, estes componentes se manifestam, por exemplo, quando autores submetem artigos a revistas, completamente dentro do foco e escopo editorial, e ainda assim, recebem como retorno uma negativa do editor-chefe, antes da avaliação, afirmando que o assunto de sua pesquisa não é prioridade.

0 caminho adotado neste artigo para mapear o desempenho dos assuntos frente ao Qualis foi a comparação das DTN com a classificação dos periódicos em que se encontram alocados. Desta análise, podem incorrer insights sobre os temas que obtêm acesso mais frequente aos estratos superiores e inferiores deste sistema de classificação. A partir disto, encontrou-se o panorama verificável na Figura 4, que logo em seguida, é complementado pela Tabela 4, contendo uma discriminação mais precisa dos dados.

Figura 4 - Estrato Qualis Capes versus Doenças Tropicais Negligenciadas, no universo analisado: 2015 a 2018

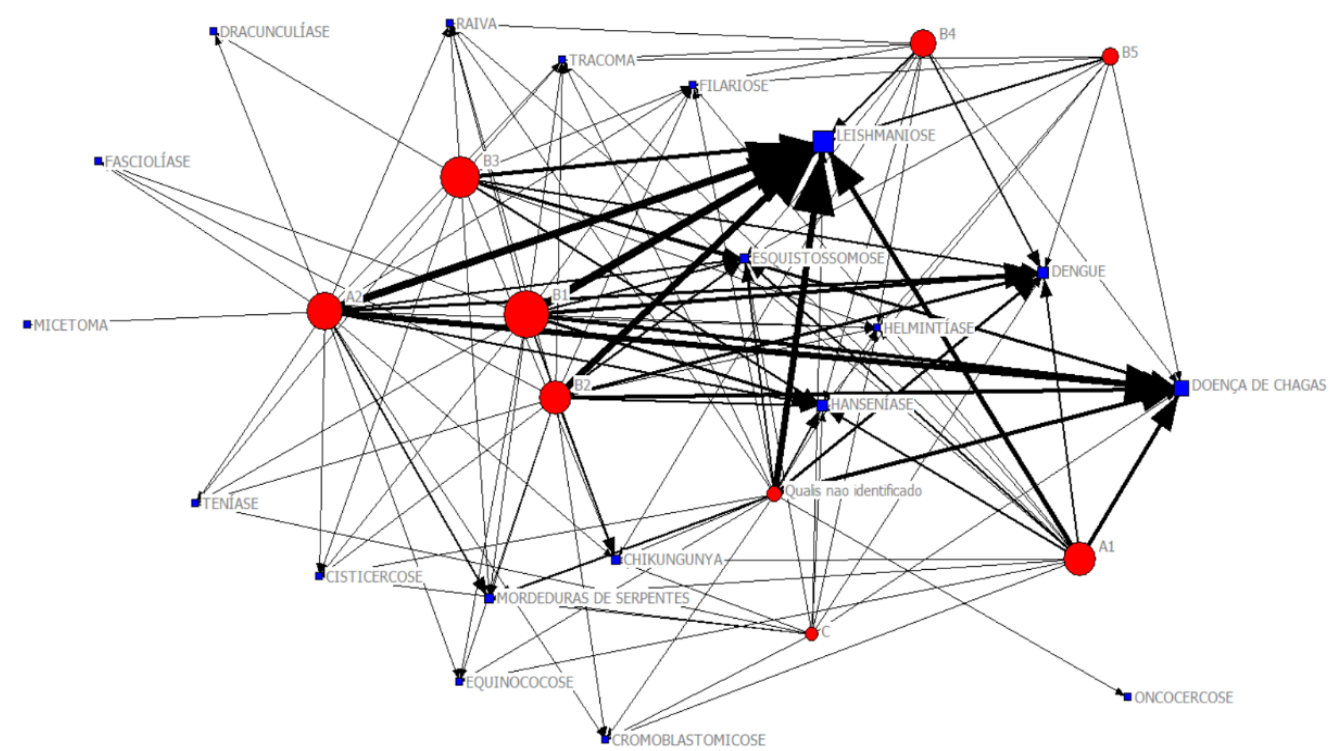

Fonte: Dados da pesquisa (2020) 


\begin{tabular}{|c|c|c|c|c|c|c|c|c|c|c|}
\hline ヨSOJYJJ0JNO & & O. & $\begin{array}{l}0 \\
0 \\
0\end{array}$ & $\begin{array}{l}0 \\
0 \\
0\end{array}$ & ㅇ. & 잉 & O. & ㅇ. & ㅇ․ & $\begin{array}{l}0 \\
8 \\
8\end{array}$ \\
\hline 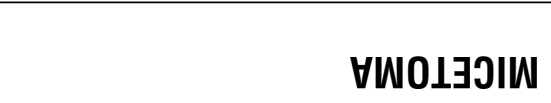 & & $0^{\circ}$ & $\begin{array}{l} \\
8 \\
8\end{array}$ & $\begin{array}{l}0 \\
0\end{array}$ & $0^{\circ}$ & $\begin{array}{l}0 \\
0\end{array}$ & $\begin{array}{l}0 \\
0^{-}\end{array}$ & $\stackrel{0}{0}$ & $\begin{array}{l}0 \\
0^{\prime}\end{array}$ & $0^{\circ}$ \\
\hline 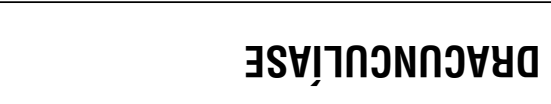 & & $0^{\circ}$ & $\begin{array}{l}0 \\
0 \\
10 \\
10\end{array}$ & $\stackrel{O}{0}^{\prime}$ & O. & $\begin{array}{l}0 \\
0 \\
10\end{array}$ & $\begin{array}{l}0 \\
0^{-}\end{array}$ & $\begin{array}{l}0 \\
0^{\prime}\end{array}$ & $\begin{array}{l}0 \\
0^{\prime}\end{array}$ & $0_{0}^{\circ}$ \\
\hline ヨSHI!10IJSHJ & & $0^{\circ}$ & \begin{tabular}{|l|}
$m$ \\
$m$ \\
$m$
\end{tabular} & \begin{tabular}{|l|} 
\\
$\tilde{m}$ \\
$m$
\end{tabular} & $\begin{array}{l}m \\
m \\
m \\
m\end{array}$ & $O^{\circ}$ & $\begin{array}{l}0 \\
0\end{array}$ & $\begin{array}{l}0 \\
0^{\prime}\end{array}$ & $\begin{array}{l}0 \\
0^{\prime}\end{array}$ & $0^{\circ}$ \\
\hline ヨS0J0J0NInOJ & & $F$ & $\begin{array}{l}m \\
\tilde{m} \\
m\end{array}$ & $\begin{array}{l}\tilde{N} \\
\tilde{N}\end{array}$ & $F$ & $0^{\circ}$ & O. & $\begin{array}{l}0 \\
0^{-}\end{array}$ & $0^{\prime}$ & $\tilde{N}$ \\
\hline 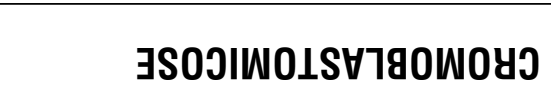 & & $\begin{array}{l}0 \\
0 \\
0\end{array}$ & $\begin{array}{l}0 \\
8 \\
0\end{array}$ & O & 웅 & $0^{\circ}$ & $\begin{array}{l}0 \\
0\end{array}$ & $\stackrel{0}{\circ}$ & $\begin{array}{l}\text { 음 } \\
0^{\circ}\end{array}$ & 웅 \\
\hline$\exists S \forall ! ̣ N \exists I$ & & $O^{\circ}$ & $\begin{array}{l}m \\
\infty\end{array}$ & $\frac{\bar{q}}{\bar{q}}$ & $\tilde{m}^{m}$ & $\begin{array}{l}\infty \\
\infty^{\circ}\end{array}$ & $O^{\circ}$ & $O^{\circ}$ & $\begin{array}{l}m \\
\infty\end{array}$ & O. \\
\hline$\forall$ BNity & & $\begin{array}{l}m \\
m \\
m\end{array}$ & $\begin{array}{l}m \\
m \\
m\end{array}$ & $\hat{\tilde{N}}$ & $\stackrel{\circ}{\circ}$ & $\widehat{0}$ & $\hat{\sigma}^{-}$ & $O_{0}^{\prime}$ & $O_{0}^{\circ}$ & $m^{m}$ \\
\hline ヨSOJYヨગIISI0 & & $0^{\circ}$ & $\begin{array}{l} \\
\\
0 \\
\end{array}$ & $\begin{array}{l}0 \\
0 \\
0 \\
\end{array}$ & م. & $\begin{array}{l}0 \\
\stackrel{0}{N}\end{array}$ & O & $\begin{array}{l}0 \\
0\end{array}$ & $\begin{array}{l} \\
\\
10\end{array}$ & '요 \\
\hline 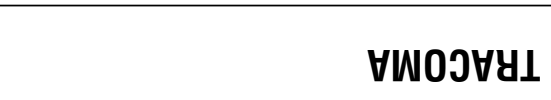 & $\stackrel{2}{0}$ & $\begin{array}{l}\infty \\
\text { ले }^{-}\end{array}$ & $\hat{N}$ & $\begin{array}{l} \\
50 \\
5\end{array}$ & $\begin{array}{l}\text { क } \\
0 \\
\sim\end{array}$ & $\hat{N}$ & 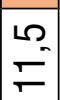 & $\begin{array}{l}0 \Omega \\
\Sigma \\
\end{array}$ & $\begin{array}{l}0 \\
0\end{array}$ & $\begin{array}{l}\nabla \\
\stackrel{5}{\circ}\end{array}$ \\
\hline 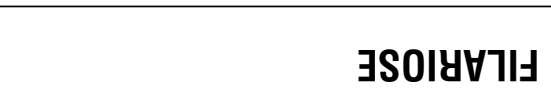 & & $\begin{array}{l}\sigma^{\prime} \\
\sigma^{\prime}\end{array}$ & $\stackrel{\infty}{\sigma}$ & \begin{tabular}{|l|} 
\\
0 \\
0 \\
\end{tabular} & $\bar{\sigma}$ & $\begin{array}{l}\nabla^{\prime} \\
\sigma^{\prime}\end{array}$ & $\bar{\sim}$ & $\begin{array}{l}m \\
\stackrel{m}{\sim} \\
\end{array}$ & $\begin{array}{l}0 \\
0\end{array}$ & $\begin{array}{l}\infty \\
\stackrel{N}{N}\end{array}$ \\
\hline 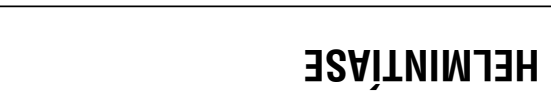 & & $\bar{N}$ & \begin{tabular}{l}
$m$ \\
\multirow{I}{*}{}
\end{tabular} & 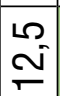 & $\begin{array}{l}0 \\
\sigma \\
\end{array}$ & $\stackrel{\Omega}{\stackrel{s}{2}}$ & $\stackrel{\infty}{-}$ & $\begin{array}{l}\mathscr{0} \\
\dot{m}^{\prime}\end{array}$ & $\begin{array}{l}\vec{T} \\
\end{array}$ & 요 \\
\hline 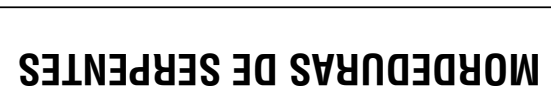 & & $\nabla_{-}$ & $\begin{array}{l}\text { s. } \\
\text { ñ } \\
\text { m. }\end{array}$ & \begin{tabular}{|l|}
\multirow{\sigma}{\sigma}{} \\
$\sigma^{\prime}$
\end{tabular} & $\ddot{\infty}$ & \begin{tabular}{|l|l|} 
\\
\\
\end{tabular} & O. & O. & $\nabla_{-}$ & $\overline{8}$ \\
\hline 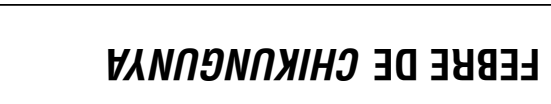 & & $\begin{array}{l}m \\
N \\
N\end{array}$ & $\begin{array}{l}m \\
N \\
\frac{m}{2}\end{array}$ & $\begin{array}{l}0 \\
\stackrel{\sim}{\sim}\end{array}$ & $\hat{0}$ & $\begin{array}{l}m \\
m^{\prime}\end{array}$ & $\stackrel{\circ}{\circ}$ & $O_{0}^{\prime}$ & $\stackrel{m}{-}$ & $\stackrel{0}{\simeq}$ \\
\hline בSOWOSSOISINOS & & $\begin{array}{l}\dot{J} \\
\stackrel{\sim}{\sim}\end{array}$ & $\bar{\AA}$ & $\begin{array}{l} \\
\infty^{\circ} \\
\end{array}$ & $\begin{array}{l}\infty \\
\omega^{\circ} \\
\end{array}$ & $\bar{\sigma}$ & $\tilde{m}^{m}$ & $\sigma_{-}$ & $\nabla_{-}$ & $\hat{N}$ \\
\hline ヨSHI!NヨSNHH & & \begin{tabular}{l}
\multirow{J}{*}{} \\
$\tilde{\sigma}$
\end{tabular} & $\begin{array}{l}0 \\
\text { 두 }\end{array}$ & \begin{tabular}{|l|}
$\infty$ \\
$\sigma^{-}$ \\
\end{tabular} & $\hat{0}$ & $\begin{array}{l}0 \\
150 \\
5\end{array}$ & $\stackrel{\nabla}{\sim}$ & $\stackrel{\circ}{\circ}$ & $\begin{array}{l}\infty \\
0^{\prime}\end{array}$ & م) \\
\hline ヨกפNJ0 & & $\begin{array}{l}\infty \\
0 \\
0\end{array}$ & $\begin{array}{l}\nabla^{\prime} \\
\nabla^{\prime}\end{array}$ & 吾 & $\tilde{J}^{-}$ & L & $\begin{array}{l}\infty \\
10 \\
10\end{array}$ & $\stackrel{m}{\sigma}$ & $\sigma_{-}$ & $\sigma^{\circ}$ \\
\hline 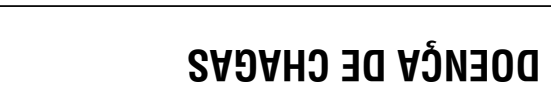 & & $\begin{array}{l}\infty \\
0 \\
0\end{array}$ & $\begin{array}{l}\sim \\
\stackrel{N}{\sim}\end{array}$ & $\begin{array}{l}\nabla_{-} \\
\underset{\nabla}{+}\end{array}$ & $\begin{array}{l}m \\
m \\
m\end{array}$ & क & $\stackrel{\infty}{-\infty}$ & ָ & $\tilde{\sigma}^{\prime}$ & $\begin{array}{l}\infty \\
0 \\
0\end{array}$ \\
\hline ヨSOINHWHSIJר & & 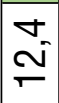 & $\begin{array}{l} \\
\\
\end{array}$ & $\begin{array}{l}\dot{D}^{\prime} \\
\stackrel{\sim}{*}\end{array}$ & $\bar{\sigma}$ & $\begin{array}{l}\nabla^{-} \\
\infty\end{array}$ & $\tilde{\sim}$ & $\bar{\sim}$ & 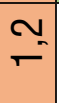 & $\stackrel{n}{n}$ \\
\hline 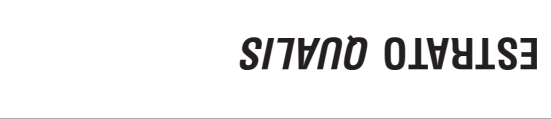 & & $\bar{z}$ & $\stackrel{\sim}{\gtrless}$ & $\bar{\infty}$ & $\widetilde{\infty}$ & $\mathscr{\infty}$ & 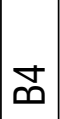 & 宓 & 0 & 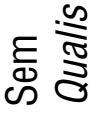 \\
\hline
\end{tabular}


Na Figura 4 apresentam-se as informações a partir de um grafo e na Tabela 4, os dados são representados conforme sua distribuição percentual. Os valores grifados em verde apontam para os índices de maior destaque, enquanto os em vermelho, refletem os percentuais menores. Nota-se que os assuntos mais proeminentes no Qualis A1 são: hanseníase (17,4\%), febre de Chikungunya (17,3\%) e doença de Chagas (16,8\%). No A2, micetoma (100\%), cromoblastomicose (60\%) e dracunculíase (50\%), porém, este grupo, com exceção de cromoblastomicose, possui um quantitativo ínfimo de produções, o que desfavorece a análise comparativa. Na categoria "sem Qualis", os destaques são: oncocercose (100\%), também pouco representativa no cômputo geral de artigos, mordeduras de serpentes $(30,1 \%)$ e filariose $(29,8 \%)$.

Quando se analisa cada DTN individualmente frente ao percentual do Qualis, observa-se que as produções em micetoma (100\%), cromoblastomicose (70\%), dracunculíase (50\%), doença de Chagas (41\%), febre de Chikungunya (34,6\%) e hanseníase $(34,4 \%)$ são os itens que obtiveram melhor desempenho no Qualis A, somando-se o $A 1$ ao $A 2$.

Ao considerarem-se apenas as doenças com quantidade igual ou superior a uma dezena de artigos, com o propósito de alcançar uma base mais representativa para estratificação percentual, vê-se o papel destacado da cromoblastomicose, com três artigos na Plos Neglected Tropical Diseases (A2), três na Frontiers in Microbiology (A2) e um no Clinical Microbiology Reviews (A1). Ressalta-se que a cromoblastomicose é uma doença de pele encontrada em populações rurais de regiões tropicais e subtropicais, sendo o Brasil um país com áreas endêmicas (GOMES et al., 2016). Em seguida, assumindo um espaço importante nos estratos mais elevados, figura a doença de Chagas, com 41 artigos na Plos Neglected Tropical Diseases (A2), 25 na Plos One (A2), 24 na Parasites \& Vectors (A2) e 17 na Frontiers in Immunology (A1). A doença de Chagas, também conhecida como tripanossomíase americana, segundo a OMS é uma doença potencialmente fatal causada pelo parasita protozoário Trypanosoma cruzi (T. cruzi). A doença leva o nome de Carlos Justiniano Chagas, célebre médico brasileiro que descobriu a doença em 1909 (OMS, 2019).

Em relação à categoria "sem Qualis", percebe-se que oncocercose (100\%), mordedura de serpentes $(30,1 \%)$, filariose $(29,8 \%)$, equinococose $(22,2 \%)$ e dengue $(19,4 \%)$ são as doenças com mais artigos, percentualmente, não estratificados. Todavia, pontua-se a baixa ocorrência de artigos sobre oncocercose e equinococose. Outro dado importante, é que muitos pesquisadores do universo pelo qual se obteve a produção científica, especialmente os da Fiocruz, parcela mais representativa, possuem formações distintas dos campos tradicionais da Saúde e publicam bastante em suas 
áreas-mães, como por exemplo, Educação, História, Ciência da Informação, Ciência da Computação, Química, Biologia, dentre outras áreas que possuem sua própria tabela de classificação Qualis. Com isto, é possível que muitos artigos destes pesquisadores possam ter sido deslocados para a zona sem Qualis devido ao parâmetro adotado neste estudo ter sido "Medicina II". Tais pressupostos poderão ser confirmados em estudos futuros que contemplem estas peculiaridades.

Subsequentemente, analisaram-se os resultados do Qualis, apontando algumas tendências de crescimento com base em linearidade, estabelecendo previsões para 2019 (Figura 5), conforme os procedimentos descritos na seção metodológica desse artigo.

Figura 5: Qualis Capes, ano a ano, com parcial e previsão 2019, no universo selecionado (2015 a 2018 consolidados e 2019 tendências)

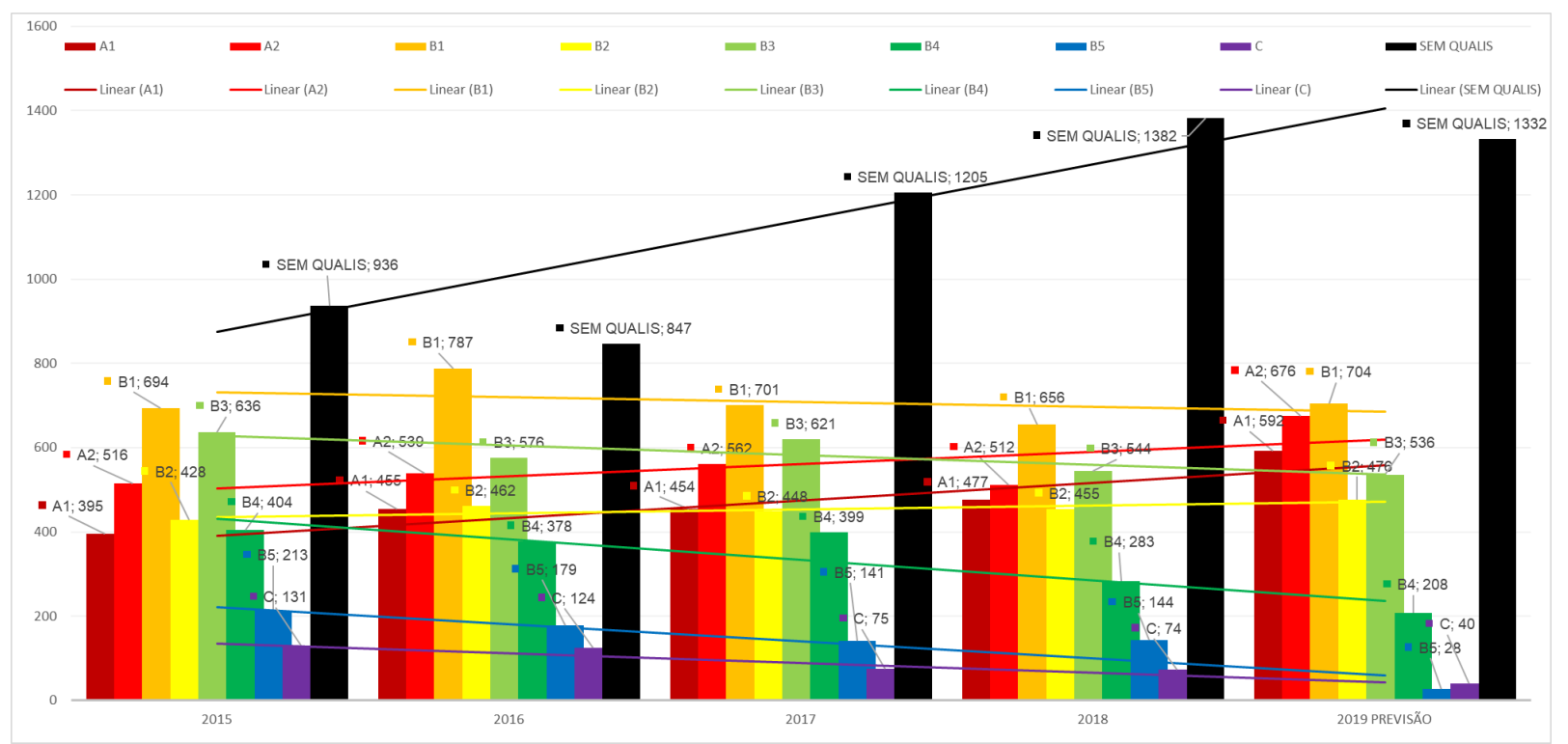

\begin{tabular}{|l|l|l|l|l|l|r|r|r|r|}
\hline \multicolumn{1}{|c|}{ Ano } & \multicolumn{10}{|c|}{ Qualis Capes } \\
\hline & A1 & A2 & B1 & B2 & B3 & B4 & B5 & C & Sem Qualis \\
\hline 2015 & 395 & 516 & 694 & 428 & 636 & 404 & 213 & 131 & 936 \\
\hline 2016 & 455 & 539 & 787 & 462 & 576 & 378 & 179 & 124 & 847 \\
\hline 2017 & 454 & 562 & 701 & 448 & 621 & 399 & 141 & 75 & 1205 \\
\hline 2018 & 477 & 512 & 656 & 455 & 544 & 283 & 144 & 74 & 1382 \\
\hline 2019 Parcial & 148 & 169 & 176 & 119 & 134 & 52 & 7 & 10 & 333 \\
\hline 2019 Previsão & 592 & 676 & 704 & 476 & 536 & 208 & 28 & 40 & 1332 \\
\hline
\end{tabular}

Fonte: Dados da pesquisa (2020)

Destarte, verificou-se a tendência de crescimento na produção "sem Qualis", provavelmente, fruto de uma alta na produção de artigos dos pesquisadores que publicam e atuam fora da Medicina Il e têm suas 
produções estratificadas nos Qualis específicos de suas áreas. No mais, enxerga-se tendência favorável nos estratos superiores $A 1$ e $A 2$, o que sugere duas frentes principais de análise: a primeira, referese ao compromisso dos pesquisadores de buscarem veículos mais qualificados, objetivando melhores resultados frente às instâncias avaliativas; e a segunda, concernente ao possível aprofundamento da qualidade das pesquisas, que torna possível ao pesquisador pleitear periódicos mais qualificados para a publicação dos seus papers. Ainda com tendência positiva, verifica-se o bom desempenho do estrato $B 2$.

Nos destaques negativos, se vê tendência regressiva para os estratos $B 4, B 5$ e $C$, em seguida, $B 3$ e $B 1$ próximos da estabilização, o que numa perspectiva conjuntural, aponta para a normalidade do sistema, tendo em vista que há tendência positiva nos estratos de maior qualidade, negativa nos de qualidade inferior e estabilização na zona neutra, sendo o crescimento dos "sem Qualis" a distorção.

\section{CONCLUSÕES}

Este artigo objetivou analisar o Qualis Capes e os periódicos científicos frente à produção de artigos em DTN, correlacionando-os com as doenças especificadas pela OMS. Com isto, notou-se 0 destaque das publicações Plos One, Ciência \& Saúde Coletiva, Plos Neglected Tropical Diseases, Revista da SBMT, Memórias do IOC e Cadernos de Saúde Pública, demonstrando que apesar da grande força das publicações internacionais, os periódicos nacionais internacionalizados possuem um espaço privilegiado na predileção dos pesquisadores brasileiros.

Em relação aos núcleos temáticos, viu-se que a doença leishmaniose está presente nas principais relações, sendo o principal assunto dentre as DTN em veículos nacionais e internacionais, como por exemplo, na Plos Neglected Diseases, Plos One, Parasites \& Vectors, Revista da SBMT, Memórias do $10 C$ e Frontiers in Immunology. Em relação às doenças com ocorrência significativa na produção científica com maior inserção percentual no Qualis A, destacaram-se cromoblastomicose e doença de Chagas, impulsionadas pelos periódicos Plos Neglected Tropical Diseases (A2), Frontiers in Microbiology (A2), Plos One (A2), Parasites \& Vectors (A2) e Frontiers in Immunology (A1).

No mais, notou-se que a projeção de distribuição dos estratos Qualis apontam para uma normalidade sistêmica, onde se vê tendência positiva para os estratos de maior qualidade, negativa para os de qualidade inferior e estabilização na zona neutra, sendo o crescimento dos "sem Qualis" a distorção. Com isto, infere-se que há um esforço deste conjunto de pesquisadores rumo aos padrões de qualidade institucionalmente estabelecidos. 
Por fim, apresentam-se como limitações do estudo alguns critérios que serão reformulados na continuação da pesquisa, como por exemplo, a consideração apenas dos pesquisadores doutores da Fiocruz e a generalização do Qualis de Medicina II para todo o conjunto, todavia, expressa-se que tais decisões asseguraram a viabilidade e o custo-benefício da pesquisa com a manutenção da devida qualidade.

\section{AGRADECIMENTOS}

Ao Cientista de Dados e Prof. Dr. Ricardo Barros Sampaio pela cessão de dados de pesquisa do projeto e-Lattes e ao Laboratório Otlet $\mathrm{Cl}$ da Universidade Federal de Pernambuco. 0 presente trabalho foi realizado com apoio da Coordenação de Aperfeiçoamento de Pessoal de Nível Superior, Brasil (CAPES) - Bolsa de Doutorado.

\section{REFERÊNCIAS}

BORGATTI, S. P.; EVERETT, M. G.; FREEMAN, L. C. UCINET for windows: software for social network analysis. Harvard: Analytic Technologies, 2002.

BRASIL. Coordenação de Aperfeiçoamento de Pessoal de Nível Superior. Qualis Periódicos e classificação de produção intelectual. 2014. Disponível em: https://www.capes.gov.br/avaliacao/ instrumentos-de-apoio/qualis-periodicos-e-classificacao-de-producao-intelectual. Acesso em: 01 mai. 2020.

BRASIL. Estratégia Nacional de Ciência, Tecnologia e Inovação - 2016-2022. Brasília: Ministério da Ciência, Tecnologia, Inovações e Comunicações, 2016. 132 p. Disponível em: https://portal.insa.gov. br/images/documentos-oficiais/ENCTI-MCTIC-2016-2022.pdf. Acesso em: 01 fev. 2020.

BRASIL. Fundação Oswaldo Cruz. Pesquisa e Ensino. 2019. Disponível em: https://portal.fiocruz.br/ pesquisa-e-ensino. Acesso em: 30 abr. 2019.

CAMARG0, E. P. Doenças tropicais. Estudos avançados, v. 22, n. 64, p. 95-110, 2008. Disponível em: http://www.producao.usp.br/handle/BDPI/11791. Acesso em: 02 mar. 2020.

COIMBRA JúNIOR. C. E. A. Produção científica e impacto em saúde coletiva. Cad. Saúde Pública, Rio de Janeiro, v. 20, n.4, p. 878-879, jul./ago., 2004. Disponível em: http://www.scielo.br/pdf/csp/ v20n4/01.pdf. Acesso em: 25 ago. 2019.

CROSS, D; THOMSON, S; SINCLAIR, A. Research in Brazil: A report for CAPES by Clarivate Analytics. Philadelphia: Clarivate Analytics, 2017. 73 p. Disponível em: https://www.capes.gov.br/ images/stories/download/diversos/17012018-CAPES-InCitesReport-Final.pdf. Acesso em: 20 abr. 2019. 
DELIBERATO, P. C. P. Modelos de atuação preventiva em saúde: relação entre saúde e doença.

Revista de Atenção à Saúde (antiga Rev. Bras. Ciên. Saúde), v. 1, n. 1, 2003. Disponível em: http:// seer.uscs.edu.br/index.php/revista_ciencias_saude/article/viewFile/492/338. Acesso em: 20 dez. 2019.

FORATTINI, O. P. O Brasil e a medicina tropical. Rev. Saúde Pública, São Paulo, v. 31, n. 2, Abr. 1997. Disponível em: http://www.scielo.br/scielo.php?script=sci arttext\&pid=S003489101997000200002\&Ing=en\&nrm=iso. Acesso em: 10 dez. 2019.

GOMES, R. R. et al. Molecular epidemiology of agents of human chromoblastomycosis in Brazil with the description of two novel species. PLoS Negl Trop Dis, v. 10, n. 11, e0005102.

GONZÁLEZ-ALCAIDE, G. et al. Evolution of coauthorship networks: worldwide scientific production on leishmaniasis. Revista da Sociedade Brasileira de Medicina Tropical, Uberaba, v. 46, n. 6, p. 719727, dez. 2013. Disponível em: http://dx.doi.org/10.1590/0037-8682-0207-2013. Acesso em: 14 fev. 2020.

GONZÁLEZ-ALCAIDE, G. et al. Scientific authorships and collaboration network analysis on Chagas disease: papers indexed in PubMed (1940-2009). Revista do Instituto de Medicina Tropical de São Paulo, São Paulo, v. 54, n. 4, p. 219-228, jul./ago. 2012. Disponível em: http://dx.doi.org/10.1590/S003646652012000400007. Acesso em: 14 fev. 2020.

GUIMARÃES, Y. V. Animais como sentinelas em doenças infecciosas. 2015. 21 f. TCC (Graduação) - Curso de Medicina Veterinária, Universidade de Brasília, Brasília, 2015. Disponível em: http://bdm. unb.br/bitstream/10483/13326/1/2015_YasminVilelaGuimaraes.pdf. Acesso em: 01 mar. 2020.

HJØRLAND, B. Domain analysis. In: HJØRLAND, Birger; GNOLI, Claudio (Ed.). Encyclopedia of Knowledge Organization. 1.3, [s.l.]: Isko, 2017. p. 1-2. Disponível em: https://www.isko.org/cyclo/ domain_analysis.htm. Acesso em: 15 jul. 2019.

HJØRLAND, B. Domain analysis in information science: eleven approaches-traditional as well as innovative. Journal of documentation, v. 58, n. 4, p. 422-462. 2002.

LOBATO, F. Qualis único é retrocesso, avalia o editor Kenneth Camargo, da Abrasco. 2019. Entrevista cedida por Keneth Camargo Junior para o Portal de Periódicos Fiocruz. Disponível em: https://periodicos.fiocruz.br/pt-br/content/qualis-\%C3\%BAnico-\%C3\%A9-retrocesso-avalia-0-editorkenneth-camargo-da-abrasco. Acesso em: 09 mar. 2020.

MENA-CHALCO, J. P.; CESAR JUNIOR, R. M. ScriptLattes: an open-source knowledge extraction system from the Lattes platform. Journal of the Brazilian Computer Society, Porto Alegre, v. 4, n. 15 , p.31-39, 2009. Disponível em: http://www.scielo.br/pdf/jbcos/v15n4/04.pdf. Acesso em: 5 jun. 2019.

MERTON, R. K. The Mathew effect in science. Science, Washington, v. 159, n. 3810, p. 56-63, Jan. 1968.

MOURA, M. Universidades públicas respondem por mais de $95 \%$ da produção científica do Brasil. Ciência na Rua. [s.I.], p. 1-3. 11 abr. 2019. Disponível em: http://ciencianarua.net/universidadespublicas-respondem-por-mais-de-95-da-producao-cientifica-do-brasil/. Acesso em: 20 abr. 2019. 
ORGANIZAÇÃO MUNDIAL DA SAÚDE (OMS). Chagas disease (American trypanosomiasis): what is Chagas disease? 2019. Disponível em: https://www.who.int/chagas/disease/en/. Acesso em: 01 dez. 2019.

ORGANIZAÇÃO MUNDIAL DA SAÚDE (OMS). Neglected tropical diseases. 2017. Disponível em: http:// www.who.int/neglected_diseases/diseases/en/. Acesso em: 24 mar. 2020.

RIZZOTTO, M. L. F.; COSTA, A. M.; LOBATO, L. de. V. C. Os novos critérios da Capes para classificação dos periódicos e a repercussão no campo da saúde coletiva. Saúde em debate, Rio de Janeiro, v. 43, n. 122, p. 649-656, Set. 2019. Disponível em: http://www.scielo.br/scielo. php?script=sci_arttext\&pid=S0103-11042019000300649\&lng=en\&nrm=iso. Acesso em: 24 mar. 2020.

ROSA, D. W. Avaliação da atividade leishmanicida da perezona incorporada em gel em modelo animal de leishmaniose cutânea. 2017. 101 f. Dissertação (Mestrado) - Curso de Farmácia, Programa de Pós-graduação em Farmácia, Universidade Federal de Santa Catarina, Florianópolis, SC, 2017. Disponível em: https://repositorio.ufsc.br/bitstream/handle/123456789/180250/347964. pdf?sequence=1\&isAllowed=y. Acesso em: 24 mar. 2020.

SAMPAIO, R. B. As estruturas globais e regionais do campo de pesquisa, desenvolvimento e inovação das doenças negligenciadas leishmaniose e tuberculose sob a ótica das redes complexas. 2015. 219 f. Tese (Doutorado) - Curso de Ciência da Informação, Faculdade de Ciência da Informação, Universidade de Brasília, Brasília, 2015. Disponível em: http://repositorio.unb.br/ handle/10482/19126. Acesso em: 01 abr. 2016.

SOBRAL, N. V.; SILVA, F. M.; DUARTE, Z. Perfil da produção científica em Medicina Tropical na América Latina: análise do termo "Tropical Medicine" na Web of Science. Em Questão, Porto Alegre, v. 23, p.31-49, 27 jan. 2017. Faculdade de Biblioteconomia Comunicacao. Disponível em: https://seer. ufrgs.br/index.php/EmQuestao/article/view/6809. Acesso em: 01 abr. 2019.

UNIFESP. Universidades públicas realizam mais de $\mathbf{9 5 \%}$ da ciência no Brasil. 2019. Disponível em: https://www.unifesp.br/noticias-anteriores/item/3799-universidades-publicas-realizam-mais-de-95da-ciencia-no-brasil. Acesso em: 20 abr. 2019.

WASSERMAN, S.; FAUST, K. Social network analysis: methods and applications. Cambridge: Cambridge University Press, 1994.

WORBOYS, M. Tropical diseases. In: BYNUM, W F; PORTER, Roy (Ed.). Companion Encyclopedia of the History of Medicine. Londres: Routledge, 2013. Cap. 24. On-line. 